\title{
KINETICS OF ELIMINATION OF GLUCOSE FROM THE BLOOD DURING AND AFTER A CONTINUOUS INTRAVENOUS INJECTION
}

\author{
BY S. G. JOKIPII AND OSMO TURPEINEN 1 \\ (From the Third Department of Medicine, Helsinki University, Helsinki, Finland)
}

(Submitted for publication May 12, 1953; accepted November 4, 1953)

The glucose tolerance tests in routine clinical use are predominantly of the oral type, in which the sugar is administered by mouth. Such tests, however, suffer from the disadvantage that the rate of intestinal absorption has an effect on the results, and its variations may entirely overshadow any possible changes in carbohydrate metabolism. This difficulty may, of course, be obviated by administering the sugar by vein, and intravenous tolerance tests have actually been used by many workers (1-8). ${ }^{2}$ In these tests the sugar has generally been given as a single rapid injection. While such a method undoubtedly gives useful information, it can hardly be regarded as a quite physiological one. A more natural procedure would be to inject glucose slowly over a long period of time, thus imitating the normal process of absorption. So far, tolerance tests of this type have apparently received scant attention. As it seemed that this method might provide additional information not easily obtainable from the tests based on a single rapid injection, we decided to carry out the following experiments, in which glucose was administered as a continuous injection of constant rate and of relatively long duration.

\section{METHODS}

Our subjects were hospitalized patients. During the experiments they were in the postabsorptive state. Approximately isotonic (5 to 6 per cent) glucose solutions in distilled water were used, and their glucose content was determined by polarimetry.

The sterilized glucose solution was run by gravity from the infusion apparatus into the cubital vein. The rate of infusion was kept as nearly constant as possible. It was checked at frequent intervals by means of a dropcounting device and readjusted if necessary. The infu-

\footnotetext{
1 Present Address: Department of Physiology, Veterinary College, Helsinki, Finland.

2 This is not meant to be a complete bibliography. Tunbridge and Allibone (3) list no less than 37 publications dealing with intravenous glucose tolerance tests.
}

sion rates ranged from 155 to $630 \mathrm{mg}$. of glucose per minute in different experiments. In a typical experiment the infusion lasted 60 minutes. The blood sugar of the subjects was determined in samples of capillary blood by means of the Hagedorn-Jensen method (9), and the samples were generally drawn $10,20,30,40,50$, and 60 minutes after the start of the infusion and in about onehalf of the experiments also 30 and 60 minutes after the end of the infusion. Urine samples were collected at the end of the infusion and often also 30 and 60 minutes later. The subjects remained recumbent during the whole experiment.

\section{RESULTS}

\section{Mean blood glucose curve during the infusion}

The above-described glucose tolerance tests were carried out in some thirty patients in all. After the exclusion of diabetics and a few other patients suffering from diseases known to affect carbohydrate metabolism, twenty-four patients remained whose carbohydrate metabolism was presumably normal. Their results were averaged, and the course of the mean blood glucose value during the infusion is shown in Figure 1. The mean fasting blood glucose prior to the infusion was $84.9 \mathrm{mg}$. per $\mathrm{dl}^{3}$ This initial basal value was used as the zero level, and the curve shows the mean increases above this basal value after specified time intervals. It is seen that the blood glucose initially rises rapidly, then more and more slowly and seems to be approaching a plateau at the end of the infusion. When the plateau is reached, the rate of elimination obviously equals the rate of infusion.

It is of considerable interest to examine whether the blood glucose curve shown in Figure 1 could be given some relatively simple mathematical interpretation. It is known that certain substances are removed from the blood and other compartments of the body through which they are distributed at a rate defined by a first-order reaction.

$3 \mathrm{dl} .=$ deciliter $=100 \mathrm{ml}$. 


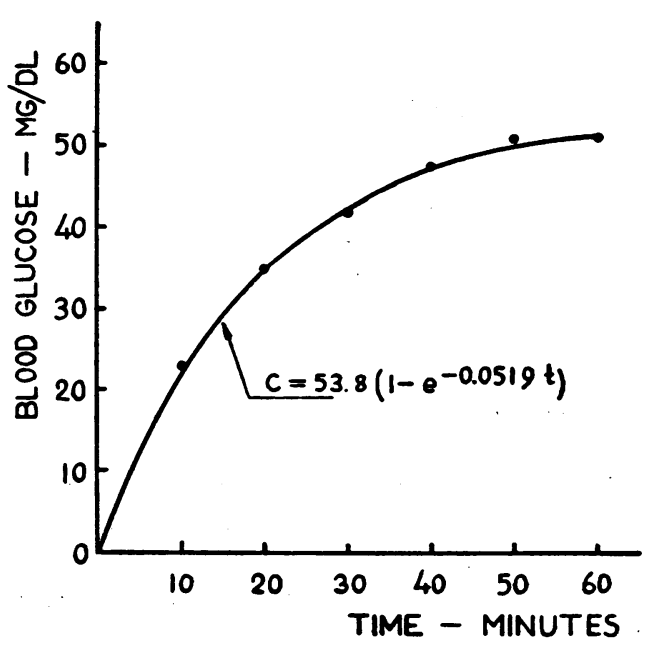

Fig. 1. The Average Course of the Blood Glucose Value in Twenty-Four Noracal Subjects during THE INFUSION

The dots indicate the arithmetic means of the observations; the curve represents the equation shown. The mean of the pre-injection blood sugar (the zero level in the graph) was $84.9 \mathrm{mg}$. per dl., that of the final blood sugar at 60 minutes $135.7 \mathrm{mg}$. per $\mathrm{dl}$., and the mean rate of infusion $297 \mathrm{mg}$. per $\mathrm{min}$.

This has been shown to be the case with, e.g., acetone (10), calcium (11), creatinine (12), xylose (13), mannitol (14), and also with glucose under experimental conditions different from ours (5).

In a first-order reaction the amount of material which is eliminated per unit of time is directly proportional to the amount that is present :

$$
-\frac{\mathrm{dq}}{\mathrm{dt}}=\mathrm{kq}
$$

( $q=$ quantity of substance in the body, $\mathrm{mg} ; \mathrm{t}=$ time, minutes; $k=$ the specific reaction-rate constant or the velocity constant of elimination, $\min .^{-1}$ ). If the volume of distribution ( $\mathrm{v} \mathrm{dl}$ ) of the substance remains constant, the situation may also be described:

$$
-\frac{\mathrm{dc}}{\mathrm{dt}}=\mathrm{kc}
$$

(c = concentration of the substance in its volume of distribution, mg. per dl.).

During the infusion the substance is simultaneously added to the body, and the rate of the concentration change caused by this obviously equals the rate of infusion ( $\rho \mathrm{mg}$. per min.) divided by the volume of distribution:

$$
+\frac{d c}{d t}=\frac{\rho}{v}
$$

Accordingly, the rate of the net increase in the concentration of the substance is as follows:

$$
\frac{\mathrm{dc}}{\mathrm{dt}}=\frac{\rho}{\mathrm{v}}-\mathrm{kc}
$$

If the concentration of the substance at the start of the infusion is zero $(c=0, t=0)$, the equation (4) gives on integration:

$$
c=\frac{\rho}{v k}\left(1-\mathrm{e}^{-k t}\right)
$$

Thus, it is evident that if a substance is injected into the blood at a constant rate (and if the firstorder reaction equation holds), the form of the concentration-time curve depends on, besides the rate of injection $(\rho)$, two characteristics of the subject : the velocity constant of elimination $(k)$ and the volume of distribution (v). If these two are known, the curve can easily be drawn. On the other hand, if the curve has been experimentally determined, the unknown quantities $\mathrm{k}$ and $\mathrm{v}$ can be derived from it.

The above reasoning should also be applicable to the glucose infusions described in this paper. In this case the concentration $c$ naturally represents the blood glucose concentration in excess above the "basal" value at the start of the infusion.

We have found that the data given in Figure 1 can quite satisfactorily be fitted with a curve corresponding to the equation (5). The fitting was done graphically on semilogarithmic paper, and the best fit was obtained with the following:

$$
c=53.8\left(1-\mathrm{e}^{-0.0519 t}\right)
$$

Thus, the velocity constant (k) was $0.0519 \mathrm{~min}^{-1}$.

The rate of elimination could, of course, also be expressed as the "period of half-life" $\left(t_{\xi}\right)$ indicating the time required for the "excess" glucose concentration to be reduced by one-half.

$$
t_{t}=\frac{0.693}{k}
$$

In this case the half-life was 13.4 minutes.

As the mean rate of infusion ( $p=297 \mathrm{mg}$. per min.) is known, the equation (5) also permits us. to compute the mean volume of distribution ( $v$ ). This was found to be 10.6 liters. As the mean 
body weight of the subjects was $62.7 \mathrm{Kg}$., the mean volume of distribution expressed in relation to body weight was 17.0 per cent. The value of $\mathrm{v}$ was also computed as percentage of the "lean body mass" (LBM). The latter, however, was not determined experimentally, but merely calculated from the predicted basal metabolic rate (15). The mean volume of distribution was found to be 20.0 per cent of the LBM.

\section{Mean blood glucose curve after the infusion}

In about one-half of the experiments the blood glucose was determined not only during the infusion but also 30 and 60 minutes after the end of the infusion. We had twelve cases in which this was done, and their mean blood glucose curve is shown in Figure 2. This is a curve which first behaves in a way very similar to that in Figure 1 and after the end of the infusion falls first steeply and then more gradually.

The rising part of the curve shown in the Figure 2 was again fitted with a curve corresponding to the equation (5) and the best fit was obtained with the following:

$$
c=55.0\left(1-\mathrm{e}^{-0.0485 t}\right)
$$

Thus, in this series the value of the velocity constant of elimination was $0.0485 \mathrm{~min}^{-1}$, which corresponds to a half-life of 14.3 minutes.

As the mean rate of infusion was $284 \mathrm{mg}$. per min., the volume of distribution was found to be 10.6 liters or 17.5 per cent of the mean body weight or 19.8 per cent of the "lean body mass."

The falling part of the blood glucose curve must obviously be computed with the assumption that the elimination also here follows the rate of a first-order reaction. We may return to the equation (4) and note that now $\rho=0$. So this equation becomes identical with the equation (2). If we designate the concentration at the zero time (the end of the infusion) as $c_{0}$, we obtain on integration:

$$
c=c_{0} e^{-k t}
$$

Our experimental data indicated that the final basal value of the blood glucose was not identical with the initial basal value. The mean blood glucose at the start of the experiment in this group was $86.5 \mathrm{mg}$. per dl., and the concentration 60 minutes after the end of the infusion was lower,

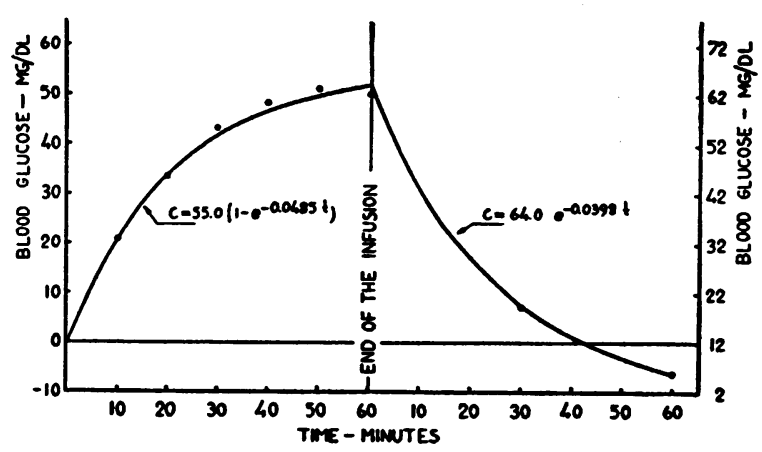

Fig. 2. The Average Course of the Blood Glucose Value in Twelve Normal Subjects during and AFTER THE INFUSION

The dots indicate the arithmetic means of the observations; the curves represent the equations shown. The mean of the pre-injection blood sugar was $86.5 \mathrm{mg}$. per dl., that of the blood sugar at the end of the infusion $136.6 \mathrm{mg}$. per dl., and 60 minutes after the end of the infusion $80.4 \mathrm{mg}$. per dl. The mean rate of infusion was $284 \mathrm{mg}$. per $\mathrm{min}$. The zeros on the axes of ordinates are: on the left-the pre-injection blood sugar; on the rightthe calculated limit (74.5 $\mathrm{mg}$. per d1.) which the blood sugar value approaches provided that the fall follows the rate of a first-order equation.

$80.4 \mathrm{mg}$. per dl. (the significance of the difference: $P=0.05$ to 0.02 ), and even this value probably did not yet represent the final basal value.

Our experimental data applied to the equation (7) gave the following:

$$
c=64.0 \mathrm{e}^{-0.0398 \mathrm{t}}
$$

According to this equation $c_{0}$ (the glucose concentration at the end of the infusion) was $64 \mathrm{mg}$. per $\mathrm{ml}$. above the final basal value. However, according to the equation $(5 b)$ this same concentration (c at 60 minutes) was only $52 \mathrm{mg}$. per dl. above the initial basal value. The final basal value must, therefore, have been $12 \mathrm{mg}$. per $\mathrm{dl}$. lower than the initial basal value. The appearance of such a phase of relative hypoglycemia in the latter part of various glucose tolerance tests is, of course, a common observation. It is also worthy of note that the velocity constant of elimination during the latter part of the experiment appeared to be somewhat smaller than during the first part." It seems

4 The statistical significance of this difference cannot be given accurately, since the values of $\mathbf{k}$ were determined through graphical fitting only. From the scatter of the blood glucose values it may, however, be estimated that the standard errors of the values of $k$ were approximately 
TABLE I

Data on blood sugar curves treated individually.

\begin{tabular}{|c|c|c|c|c|c|c|c|}
\hline $\begin{array}{c}\text { Patient } \\
\text { no. }\end{array}$ & $\begin{array}{c}\text { Age and } \\
\text { eex }\end{array}$ & Diagnosis & ms. $/$ mix. & $\underset{\min -1}{k}$ & livers & $\%$ of $B W T$ & \% of $\stackrel{v}{L} B M \ddagger^{\prime}$ \\
\hline $\begin{array}{l}6 \\
7 \\
7 * \\
9 \\
10 \\
11 \\
17 \\
45\end{array}$ & $\begin{array}{ll}29 & M \\
38 & M \\
38 & M \\
42 & F \\
54 & F \\
20 & F \\
36 & M \\
39 & M\end{array}$ & $\begin{array}{l}\text { Emphysema } \\
\text { Rheumatoid arthritis } \\
\text { Rheumatoid arthritis } \\
\text { Neurocirculatory asthenia } \\
\text { Essential hypertension } \\
\text { Schizophrenia } \\
\text { Duodenal ulcer } \\
\text { Healthy }\end{array}$ & $\begin{array}{l}293 \\
237 \\
436 \\
330 \\
628 \\
251 \\
277 \\
592\end{array}$ & $\begin{array}{l}0.065 \\
0.041 \\
0.040 \\
0.043 \\
0.050 \\
0.076 \\
0.040 \\
0.063\end{array}$ & $\begin{array}{r}11.6 \\
11.2 \\
13.0 \\
9.2 \\
11.5 \\
7.2 \\
13.8 \\
13.5\end{array}$ & $\begin{array}{l}17.6 \\
18.7 \\
21.0 \\
17.0 \\
11.2 \\
13.8 \\
20.3 \\
19.9\end{array}$ & $\begin{array}{l}19.6 \\
20.5 \\
23.5 \\
20.0 \\
19.8 \\
15.4 \\
24.5 \\
24.0\end{array}$ \\
\hline $\begin{array}{l}-19 \\
22 \\
27 \\
28 \\
29\end{array}$ & $\begin{array}{ll}-59 & - \\
26 & M \\
30 & \mathrm{~F} \\
21 & \mathrm{M} \\
33 & \mathrm{M}\end{array}$ & $\begin{array}{l}\text { Acromegaly } \\
\text { Infectious hepatitis } \\
\text { Diabetes mellitus } \\
\text { Diabetes mellitus } \\
\text { Diabetes mellitus }\end{array}$ & $\begin{array}{l}-\overline{365} \\
383 \\
233 \\
327 \\
303\end{array}$ & $\begin{array}{l}\overline{0} \overline{0.069} \\
0.031 \\
0.052 \\
0.063 \\
0.048\end{array}$ & $\begin{array}{r}-\overline{11.2} \\
13.2 \\
4.9 \\
5.1 \\
9.4\end{array}$ & $\begin{array}{r}-\overline{-}- \\
13.5 \\
18.6 \\
9.2 \\
7.3 \\
11.9\end{array}$ & $\begin{array}{r}-\overline{18.9} \\
20.5 \\
10.7 \\
7.9 \\
13.8\end{array}$ \\
\hline
\end{tabular}

* This experiment was done seven days after the previous one. During this time the patient had gained $2 \mathrm{Kg}$. $\dagger \mathrm{BW}=$ body weight.

$\mp \mathrm{LBM}=$ lean body mass.

as if the dynamic equilibrium of the glucose metabolism were somewhat changed by the test itself.

\section{Individual blood glucose curves}

It would obviously be of considerable interest to be able to analyze also the individual data. This, however, is fraught with difficulties, for the individual blood glucose-time curves frequently show irregularities rendering their analysis rather uncertain. In about one-half of the experiments we obtained curves which were regular enough to be fitted with the equation (5) with satisfactory precision. The data pertaining to these experiments are given in Table $\mathrm{I}$.

The values of $k$ found by us for individual subjects with presumably normal carbohydrate metabolism ranged from 0.040 to $0.076 \mathrm{~min}^{-1}$. In terms of the half-life these values were equivalent to a range from 17.3 to 9.1 minutes. The range of $\mathbf{v}$ was found to be from 7.2 to 13.8 liters or from 11.2 to 21.0 per cent of the body weight or from 15.4 to 24.5 per cent of the "lean body mass."

Among the tests included in Table I are a few done on subjects suffering from diseases known to affect carbohydrate metabolism. The patient No. 19 suffering from acromegaly showed fairly normal values of $k$ and $v$. The patient No. 22, a case of infectious hepatitis, had a rather low value of $\mathbf{k}$. Of considerable interest are the three dia-

0.004. Thus the difference of 0.0087 between these two values of $\mathbf{k}$ may be somewhat too small to be statistically significant. betics, Nos. 27,28 , and $29 . .^{\circ}$ Their values of $\mathbf{k}$ were well within the normal range. On the other hand, the values of $\mathrm{v}$ were strikingly low, in two patients only about one-half of the normal and in the third also obviously below the normal range.

\section{DISCUSSION}

Our experimental data are in good agreement with the hypothesis that the elimination of the exogenous glucose from the blood takes place at the rate of a first-order reaction. In view of the fact that this physiological process is generally regarded as being a rather complex phenomenon, it is of interest to note that its reaction rate is defined by such a relatively simple equation.

Two quantities of physiological significance enter this equation, $k$ and $v$. $k$, the velocity constant of elimination, is probably the best single index of the capacity of the organism to eliminate glucose from the blood and from the rest of its volume of distribution. It also has the distinct advantage of being independent of the body size. The velocity. constant $\mathrm{k}$ indicates the fraction of glucose which is eliminated per unit of time, and its dimension is the reciprocal of time, e.g., min. ${ }^{-1}$. If so preferred, the period of half-life $\left(t_{k}\right)$ can be used instead of $k$. $v$ is the total volume of the body fluids through

- All three suffered from moderately severe diabetes and were treated with protamine zinc insulin, which was withheld from them for a period of 48 hours before the test. Their pre-injection blood sugar values ranged from 216 to $347 \mathrm{mg}$. per dl. 
which the injected glucose appears to be initially distributed. The volumes of distribution are customarily expressed as percentages of the body weight, and in terms of this measure our mean value of $\mathrm{v}$ was 17.0 per cent. This is in fair agreement with the value 19 per cent reported for glucose by Dominguez (16). Very similar values have also been reported for sucrose (18 per cent) and for inulin (15.7 per cent) by McCance (17), for mannitol (18.3 per cent ) by Dominguez, Corcoran, and Page (14), and for radioactive chloride (18.9 per cent) by Moore (18).

In regard to the volume of distribution the body weight does not, however, seem to be a very satisfactory standard of reference. In our material the variation among the individual values of $\mathrm{v}$ was more effectively reduced if they were expressed in reference to the "lean body mass" (cf. Table I). A still greater uniformity might have been obtained, if experimentally determined values for LBM had been used instead of those computed from the predicted basal metabolic rates (15).

As to the meaning of the volume of distribution, it may be difficult or impossible to correlate it with any anatomically defined space. The value found by us for it is most closely in agreement with the "chloride space," which is often identified with the extracellular fluid. This could possibly be interpreted as indicating that exogenous glucose is initially distributed through extracellular fluid and in the main excluded from cells.

Perhaps a few words should also be said about the observations concerning diabetics, even though our material is very limited. In regard to the values of $\mathrm{k}$, which appeared to be normal, it should be pointed out that in the diabetic with glycosuria this constant does not have quite the same meaning as in the normal person. In the latter the elimination of glucose takes place through utilization only ${ }^{6}$ and $k$ is purely the velocity constant of utilization $\left(k_{e l}=k_{u}\right)$. In the diabetic organism glucose is eliminated through both utilization and renal excretion, and $\mathrm{k}$ is the velocity constant of this combined process $\left(k_{e l}=k_{u}+k_{e x}\right)$. The velocity constant of utilization only must obviously be smaller than the constant of the combined process. All our diabetic subjects had glycosuria, but be-

\footnotetext{
- None of our non-diabetic subjects showed glycosuria during or after the tests.
}

cause of the lack of adequate quantitative data we were not able to compute the pure velocity constant of utilization in these subjects. ${ }^{7}$ The definitely small values of $\mathrm{v}$ in the diabetics are an interesting finding for which we are not yet prepared to offer an explanation. Obviously this observation must be confirmed in more extensive experiments.

The clinical application of intravenous glucose tolerance tests, in general, seems to be in a rather unsettled state. Lack of uniformity in both technique and interpretation is prevalent. The criterion of normality most often appears to be the time interval taken for the elevated blood glucose to regain its pre-injection value (3) or some defined relatively low value (19) or, conversely, the value of blood sugar at a certain time after injection (4). It is evident that such criteria are valid under strictly defined experimental conditions only and lack more universal applicability and deeper physiological meaning. $U_{p}$ to now, only a few attempts have been made to interpret the blood glucose-time curves in a theoretically more satisfactory way. Ross (20) used a rather complicated method as he measured the surface area under the curve by a planimeter. Hamilton and Stein (21) described a method by which the blood sugar curve was "straightened out" on semilogarithmic paper and characterized by a single figure indicating the slope of the resulting straight line. However, their use of total blood sugar values instead of values indicating the excess above the basal value made their method mathematically unsound and also difficult to apply. The only adequate mathematical treatment of blood glucose curves seems to be that of Greville (5), who characterized the curves by the use of three constants: $k, C$, and $y_{0}$, of which the first is identical with our

7 In contrast to our findings, Amatuzio, Stutzman, Vanderbilt, and Nesbitt (22), whose paper appeared shortly after ours had been submitted for publication, obtained a clear-cut difference in the values of $k$ between diabetics and normal persons. This discrepancy could hardly have arisen from the differences in the technique of the test. (They used the single rapid injection method.) It may possibly be due to the fact that their patients did not receive exogenous insulin, whereas ours did. The 48-hour period of insulin deprivation imposed on our patients prior to the tests may not have been sufficiently long to permit a complete disappearance of the effect of protamine zinc insulin. 
$\mathrm{k}$, the second is definitely correlated with our $\mathrm{v}$, and the third is the "limiting value" of blood sugar. ${ }^{8}$

In this paper we have tried to treat intravenous blood glucose curves in a way which would be both mathematically defensible and physiologically meaningful. As our material is relatively limited, we have sought support for our conclusions from the work published earlier and have found it to a considerable extent. As far as we are aware, no work with a technique comparable to ours has been reported. On the other hand, a large volume of work on tests based on a single rapid injection appears in the literature. Of course, these two types of tests do not necessarily give concordant results. After the single injection the organism is exposed to unphysiologically high blood sugar concentrations (often in excess of $300 \mathrm{mg}$. per dl.), which may or may not disturb the normal course of glucose metabolism. The continuous type of injection is free from this objection.

We have examined a number of papers dealing with tolerance tests of the single injection type. Most of the reported data are in good agreement with the assumption that exogenous glucose is eliminated at the rate of a first-order reaction. The equation (7) can then be used for calculating the values of $k$. We carried out this calculation from some published material and found the following mean values: From Lennox and Bellinger (2) $: \mathrm{k}=0.037$; from Lozner, Winkler, Taylor, and Peters (4): $\mathrm{k}=0.045$; from Goldberg and Luft (6) : $k=0.063$; and from Moyer and Womack $(8): \mathrm{k}=0.044$. A "typical response" reported by Tunbridge and Allibone (3) gave: $\mathrm{k}=$ $0.054 .^{9}$ So, in spite of great differences in technique, the values of $\mathrm{k}$ calculated from this earlier material are in essential agreement with our mean value for $k, 0.052$. On the other hand, the values reported by Greville (5) are definitely lower, from 0.018 to $0.039 .^{10}$ The values of $\mathrm{v}$ are more difficult to obtain from the curves of the single injection tests. They can, however, be computed as

${ }^{8}$ Recently Amatuzio, Stutzman, Vanderbilt, and Nesbitt (22) also used the constant $\mathrm{k}$ to characterize the glucose tolerance after a single rapid intravenous injection.

9 Amatuzio, Stutzman, Vanderbilt, and Nesbitt (22) reported values of $\mathrm{k}$ ranging from 0.030 to 0.048 .

${ }_{10}$ However, the lower values of $\mathrm{k}$ were usually associated with such low values of $y_{0}$ as to cast some doubt on the physiological soundness of his interpretation. suggested by .Widmark (10) by the use of the equation: $\mathrm{v}=\frac{\mathrm{q}}{\mathrm{c}_{0}}$, in which $\mathrm{q}$ is the quantity of glucose injected and $c_{o}$ is the hypothetical concentration which would exist immediately after the injection if the steady state of diffusion were reached at once. $c_{0}$ is obtained through extrapolation from the logarithmically linear part of the curve ignoring the initial high concentrations after the injection. This method, however, is not free from objections. Through such a calculation we obtained from Lennox and Bellinger's (2) material $\mathrm{v}=10.3$ liters, from the data of Lozner, Winkler, Taylor, and Peters (4) v = 8.1 liters, and from that of Tunbridge and Allibone (3) v $=9.2$ liters, ${ }^{11}$ values which are not far from ours.

To conclude, we believe that the intravenous glucose tolerance test is a method susceptible of a theoretically sound treatment. As our experience and the evidence obtained from earlier work indicate, the elimination of exogenous glucose takes place at the rate of a first-order reaction, at least with fair approximation. From the blood glucosetime curves two constants, $\mathrm{k}$ and $\mathrm{v}$, can be derived, which characterize the course of the curve. $k$ is the specific reaction-rate constant or the velocity constant, a concept widely used in chemical kinetics, and $\mathrm{v}$ is the apparent volume through which the injected glucose is initially distributed. The values of $\mathrm{k}$ and $\mathrm{v}$ probably are in a large degree independent of the experimental details of the test, and it is evident that they can also be determined, though possibly less accurately, by means of the single injection technique. In the interest of a theoretically sound and physiologically meaningful interpretation of the glucose tolerance tests, these two constants would obviously offer definite advantages over the more or less arbitrary criteria now commonly used. Further work is certainly required to establish normal mean values and ranges for them. However, from our experiments reported here it would appear that in normal persons the mean value of $\mathrm{k}$ is approximately $0.05 \mathrm{~min}^{-1}$ (corresponding to a half-life of some 14 minutes) and that of $\mathrm{v}$ about 10 to 11 liters.

11 From the material published by Amatuzio, Stutzman, Vanderbilt, and Nesbitt (22) we obtained the mean value: $\mathrm{v}=13.7$ liters. 


\section{SUM MARY}

Glucose solutions were injected intravenously at a constant relatively slow rate during a period of 60 minutes into human subjects. The blood glucose level was determined in samples of capillary blood during and, in part of the experiments, also after the injection.

The experimental results were in good agreement with the hypothesis that the elimination of the exogenous glucose from the blood takes place at the rate of a first-order reaction. The form of the blood glucose-time curve is determined by, besides the rate of injection, two characteristics of the subject: $k$, the specific reaction-rate constant or the velocity constant of elimination, and $\dot{v}$, the apparent initial volume of distribution.

The mean value of $k$ obtained from tests with twenty-four subjects whose carbohydrate metabolism was presumably normal was $0.052 \mathrm{~min}^{-1}$. This corresponds to a period of half-life of 13.4 minutes. The mean value of $\mathrm{v}$ was 10.6 liters or 17.0 per cent of the body weight or 20.0 per cent of the estimated "lean body mass."

In three diabetic subjects the values of $\mathrm{k}$ were found to be within the normal range, but the values of $\mathrm{v}$ were strikingly low.

In the interest of a theoretically sound and physiologically meaningful interpretation of the glucose tolerance tests, the authors advocate the use of the constants $\mathrm{k}$ and $\mathrm{v}$ as the criteria of such tests.

\section{ACKNOWLEDGMENT}

The authors wish to acknowledge their indebtedness to Professor William Kerppola, the Head of the Department, for his permission to carry out these experiments and for the provision of facilities in his Department.

\section{REFERENCES}

1. Jörgensen, S., and Plum, T., On the differential diagnosis between benign and malignant glycosuria by means of intravenous injections of small quantities of grape-sugar. Acta med. Scandinav., 1923, 58, 161 .

2. Lennox, W. G., and Bellinger, M., Blood sugar. Comparison of blood sugar curves following ingestion and intravenous injection of glucose. Arch. Int. Med., 1927, 40, 182.

3. Tunbridge, R. E., and Allibone, E. C., The intravenous dextrose tolerance test. Quart. J. Med., 1940, n.s. 9, 11.

4. Lozner, E. L., Winkler, A. W., Taylor, F. H. L., and Peters, J. P., The intravenous glucose tolerance test. J. Clin. Invest., 1941, 20, 507.
5. Greville, G. D., The intravenous glucose tolerance equation. Biochem. J., 1943, 37, 17.

6. Goldberg, L., and Luft, R., A comparison of oral and intravenous dextrose tolerance tests in healthy subjects. Acta med. Scandinav., 1948, 132. 201.

7. Goldberg, L., and Luft, R., The diagnostic value of oral and intravenous dextrose tolerance tests in endocrine disorders with decreased dextrose tolerance: diabetes mellitus, thyrotoxicosis, Cushing's syndrome and acromegaly. Acta med. Scandinav., 1949, 135, 1.

8. Moyer, J. H., and Womack, C. R., Glucose tolerance: I. A comparison of 4 types of diagnostic tests in 103 control subjects and 26 patients with diabetes. Am. J. M. Sc., 1950, 219, 161.

9. Hagedorn, H. C., and Jensen, B. N., Zur Mikrobestimmung des Blutzuckers mittels Ferricyanid. Biochem. Ztschr., 1923, 135, 46.

10. Widmark, E. M. P., Studies in the concentration of indifferent narcotics in blood and tissues. Acta med. Scandinav., 1920, 52, 87.

11. Greville, G. D., Study of the blood-calcium following the intravenous injection of calcium salts. Biochem. J., 1931, 25, 1931.

12. Dominguez, R., and Pomerene, E., Studies of the renal excretion of creatinine. I. On the functional relation between the rate of output and the concentration in the plasma. J. Biol. Chem., 1934, 104, 449.

13. Dominguez, R., Goldblatt, H., and Pomerene, E., Kinetics of the excretion and utilization of xylose. Am. J. Physiol., 1937, 119, 429.

14. Dominguez, R., Corcoran, A. C., and Page, I. H., Mannitol: Kinetics of distribution, excretion, and utilization in human beings. J. Lab. \& Clin. Med., 1947, 32, 1192.

15. Behnke, A. R., An approach to $\mathrm{O}_{2}$ consumption of the "active protoplasmic mass" (APM). Federation Proc., 1952, 11, 11.

16. Dominguez, R., Kinetics of elimination, absorption and volume of distribution in the organism in Medical Physics, O. Glasser, ed., Vol. 2, Chicago, The Year Book Publishers, Inc., 1950, p. 486.

17. McCance, R. A., The effect of salt deficiency in man on the volume of the extracellular fluids, and on the composition of sweat, saliva, gastric juice and cerebrospinal fluid. J. Physiol., 1938, 92. 208.

18. Moore, F. D., Determination of total body water and solids with isotopes. Science, 1946, 104, 157.

19. Crawford, T., A standard intravenous glucose tolerance test. Arch. Dis. Childhood, 1938, 13, 69.

20. Ross, C. W., The determination of glucose tolerance. Arch. Dis. Childhood, 1938, 13, 289.

21. Hamilton, B., and Stein, A. F., The measurement of intravenous blood sugar curves. J. Lab. \& Clin. Med., 1942, 27, 491.

22. Amatuzio, D. S., Stutzman, F. L., Vanderbilt, M. J., and Nesbitt, S., Interpretation of the rapid intravenous glucose tolerance test in normal individuals and in mild diabetes mellitus. J. Clin. Invest., 1953, $32,428$. 